\title{
HALE-BOPP: WHAT MAKES A BIG COMET DIFFERENT? COMA DYNAMICS: OBSERVATIONS AND THEORY
}

\author{
MICHAEL COMBI \\ Department of Atmospheric, Oceanic and Space Sciences, University of Michigan, Ann Arbor, \\ Michigan, USA
}

(Received 10 April 2002; Accepted 25 May 2002)

\begin{abstract}
Comet Hale-Bopp was the largest comet by almost any definition, observed at least since the advent of modern observing techniques. In a more typical comet both the chemical and dynamical influences of collisional processes are limited by the short time a parcel of gas sublimated from the nucleus remains in the dense part of the coma. The resulting large size of the collisional coma in comet Hale-Bopp had important consequences on the dynamics of the coma, which in turn has important consequences on how observations are interpreted with standard models. Measured velocities of typical gas species (mostly the observed radicals) as well as dust were larger than normal comets. Conversely, velocities of superthermal atomic hydrogen were smaller than normal because of the same collisional processes. Furthermore, as a consequence, dust particles, which are dragged by the outflowing gas, were also accelerated to larger velocities. Such larger velocities are not simply an interesting curiosity in their own right, because nearly all observations of dust and gas are interpreted with models of the coma that depend directly on some measurement or assumption with regard to velocity. In this presentation both observations and theory regarding the dynamical conditions in the coma of comet Hale-Bopp are summarized.
\end{abstract}

Keywords: Comet C/1995 O1 (Hale-Bopp), comets, dynamics, molecular processes

\section{Introduction}

Because of the large size of its nucleus combined with a reasonable active area, the resulting large gas sublimation rate of comet Hale-Bopp (1995 O1) afforded a number of opportunities to learn much new about the physical nature of comets and to make some special and unique observations. Unprecedented observations of the abundances of many gas species when the comet was at large heliocentric distance revealed important clues as to the nature of the cometary activity phenomenon in general (Biver et al., 2002; Bockelée-Morvan and Crovisier, 2002; Rauer et al., presentation at IAU Colloquium 186, Tenerife, Jan. 2002). A number of observations which are normally impossible, or at least very difficult, were made possible, such as spectral identification of $\mathrm{HCO}^{+}$(Lovell et al., 1998), $\mathrm{HNC}$ (Biver et al.,1999), and $\operatorname{Ar}$ (Stern et al., 2000).

Owing to the large overall gas production rates in Hale-Bopp, the dynamics of the coma were affected in ways much more extreme than in what one would call typical, bright comets, like 1P/Halley and C/1996 B2 Hyakutake. Beyond

Earth, Moon and Planets 89: 73-90, 2002.

(C) 2002 Kluwer Academic Publishers. Printed in the Netherlands. 
being interesting in its own right, an understanding of the dynamics of the coma is critical in the analysis and interpretation of most observations of gas species in the coma. The rest of this paper will present a brief summary and examples of observations and models for coma dynamics of typical comets, as well as a more thorough discussion of observations and models for the coma dynamics in comet Hale-Bopp.

\section{Coma Dynamics for Typical Comets}

The general physics, photochemical kinetics, heating and resulting expansion of the outflowing coma are reasonably well understood. Although most theoretical model calculations of the outflow are based on an oversimplified spherically symmetric nucleus and coma, all models show that the expansion of the gas from the nucleus into the near vacuum just above the surface causes the flow to become supersonic within a fraction of the nucleus radius above the surface and therefore to become essentially radial within a couple of nucleus radii (Gombosi et al., 1986). So, while the flow will not generally be spherically symmetric in either density or velocity, the flow direction will be radial. The expansion of gas which sublimates from the porous surface of the nucleus leaves the surface with a total energy indicative of the temperature of the surface near the sublimation temperature of the water $(\sim 190 \mathrm{~K})$. Therefore, a boundary layer forms which is on the order of few collisional mean-free paths thick, whereby gas is eventually transformed to what can be described by a slightly supersonic expansion (velocity $0.25 \mathrm{~km} \mathrm{~s}^{-1}$ ) at a temperature (130-140 K) somewhat below the water ice sublimation temperature (Skorov and Rickman, 1998). The exact size of this layer is also determined somewhat by the extent of dust mass-loading (Gombosi et al., 1985). Any significant surface components to the flow (i.e., surface winds) driven by tangential pressure gradients can only develop very close to the surface, and will become radial by a few times the nucleus radius in any event. After traversing this Knudsen layer, the gas can then generally be described reasonably well using continuum equilibrium approach, i.e., hydrodynamics, except in low production rate cases (e.g., small comets at large heliocentric distance and/or on the night side).

As the gas continues to expand into $4 \pi$ steradians of practically empty space, the density falls because of conservation of mass flux as the inverse square of the distance from the center of the nucleus. Initially the gas cools rapidly, and nearly adiabatically, converting the remaining thermal energy into outwardly directed flow. In addition, the gas can cool by emission of infrared radiation to space from the rotational energy mostly of water molecules and to a lesser extent CO. However, in the inner coma the absorbing gas column for escape of IR radiation to space is large and optically thick, inhibiting the efficiency of radiative cooling (Crovisier, 1984; Bockelée-Morvan and Crovisier, 1987b). Moderated by collisions with the local hot dust, the gas can cool to very low temperatures $(<20 \mathrm{~K})$ where it 
efficiently converts the initial random thermal energy ( $190 \mathrm{~K})$ into outward directed radial motion at low temperature yielding a velocity around $0.7 \mathrm{~km} \mathrm{~s}^{-1}$ (Delsemme and Miller, 1970). As this happens, photodissociation by the solar ultraviolet slowly heats the gas because of the excess energy contained in dissociation products (Huebner et al., 1992) which is then shared by the bulk gas through molecular collisions. The added energy heats the gas and further drives the outward expansion to somewhat higher velocities.

Because of the continued expansion, however, the collisional mean-free paths become large. The gas gradually falls out of local equilibrium. In a planetary atmosphere, the boundary called the exobase is defined as the altitude where the collisional mean-free-path is equal to the local atmospheric scale height. To some degree of crude approximation the atmosphere is often considered collisional below the exobase and collisionless (i.e., molecules travelling in simple ballistic trajectories) above (Chamberlain, 1962). Similarly for comets, a collision zone radius boundary has been traditionally defined as the distance, again for a spherical coma, where the local mean-free-path is equal to the distance from the center of the nucleus. For a coma which is approximated by a very simple constant expansion velocity, $v$, a gas production rate $Q$, and a molecular collision cross section, $\sigma$, this collision zone radius, according to Whipple and Huebner (1976) is given by

$$
R_{\mathrm{coll}}=\sigma Q / 4 \pi v .
$$

In a typical bright comet with a gas production rate of a few times $10^{29}$ molecules s ${ }^{-1}$, the collision zone radius is on the order of 20,000 km. For Hale-Bopp near perihelion the collision zone radius was in excess of $10^{5} \mathrm{~km}$.

This is an oversimplified description of the actual conditions for a number of reasons. Collisions first begin to become inefficient enough so that kinetic energy is no longer efficiently transferred to rotational energy and some of what is transferred can be radiated away, so the rotational temperature begins to fall below the kinetic temperature (Crovisier, 1984; Bockelée-Morvan and Crovisier, 1987b). Because water is a polar molecule, water-water collisions have a somewhat larger cross section than those between other species and water (Crifo, 1989; Combi, 1996). As will be discussed in detail in a later section of this paper, the creation rate of secondary species with large superthermal energies is fast compared with the collisional relaxation time, even well within the collision zone radius. As a result approximately $20 \%$ of the populations of secondary species are always not yet in thermal equilibrium with the bulk of the gas. Finally, and most importantly, the main heating agent of the inner coma of comets is the excess energy imparted on the $\mathrm{H}$ atom in the main photodissociation branch of water

$$
\mathrm{H}_{2} \mathrm{O}+h v \rightarrow \mathrm{H}+\mathrm{OH} .
$$

Because of the large 18 to 1 mass ratio between water molecules and $\mathrm{H}$ atoms, it takes many collisions $(\sim 7-10)$ for a superthermal $\mathrm{H}$ atom to transfer most of its 
excess energy and to heat the coma (Ip, 1983; Marconi and Mendis, 1984; Huebner and Keady, 1984; Kitamura, 1986). Because of this, the heating of the coma via this most important mechanism begins to fall from 100\% efficiency well within the traditional definition of the collision zone radius (Combi, 1987; Bockelée-Morvan and Crovisier, 1987b; Combi and Smyth, 1988a; Ip, 1989).

The effect of all these competing mechanisms is that the outflow speed, the kinetic temperature, and their rates of increase with increasing distance from the nucleus out into the coma, all increase with the magnitude of the gas production rate. For larger gas production rates the gas densities are larger and thus the collision zone is larger, increasing the photochemical heating efficiency. Figure 1 shows the results from Bockelée-Morvan and Crovisier (1987a) of a single-iteration, combined Monte Carlo hydrodynamic calculation for a spherically symmetric coma with different gas production rates. Shown are the radial outflow velocity and gas temperature as a function of distance from the center of the nucleus for gas production rates of $10^{27}, 10^{28}, 10^{29}$ and $10^{30} \mathrm{~s}^{-1}$, for a comet at a heliocentric distance of $1 \mathrm{AU}$. These calculations also account for the opacity effects of radiative cooling. When comets are closer to the sun, the photochemical rates increase as the inverse square of the heliocentric distance, therefore, all else being equal (i.e., keeping the gas production rate constant), the heating rates increase proportionally. This by itself would tend to increase both the outflow speeds and gas kinetic temperatures. However, for a typical comet, the gas production rate itself also increases as the heliocentric distance decreases, so the combined effects of increased dissociation rate and increasing collisional thermalization efficiency compound one another.

Figures 2 and 3 show model calculations for comet Kohoutek (Combi and Smyth, 1988b) and Hyakutake (Combi et al., 1999a), respectively, for a number of different heliocentric distances. The effect of this for normal bright comets is that the outflow speed in most of the observable part of the coma $\left(10^{4}\right.$ to $10^{5} \mathrm{~km}$ from the nucleus) increases with decreasing heliocentric distance. Model calculations for comet 1P/Halley were performed to explain observed expanding $\mathrm{CN}$ shells (Ip, 1989) in Figure 4 and the widths of radio emission lines (Combi, 1989) in Figure 5a, the latter having been approximated as a power law with the velocity given by $1.1 r^{-1 / 2} \mathrm{~km} \mathrm{~s}^{-1}$, where $r$ is the heliocentric distance. For slightly weaker comets than Halley, a number of investigators have adopted an outflow speed of $0.85 r^{-1 / 2} \mathrm{~km} \mathrm{~s}^{-1}$. A more careful examination of all these observations and model results shows that the $r^{-1 / 2}$ power law is only relevant within a factor of $\sim 30 \%$ of $1 \mathrm{AU}$. For large heliocentric distances the outflow speed tends to flatten and approach the $0.6-0.7 \mathrm{~km} \mathrm{~s}^{-1}$ value which gas must have from the original water sublimation temperature in the absence of much photochemical heating. At small heliocentric distances, the outflow speed increases much faster than the $r^{-1 / 2}$ power law. Model results for the heliocentric distance variation of the outflow speed for comet Halley are shown in Figure 5b. Therefore, investigators must take great care in extrapolating the $r^{-1 / 2}$ power law to small and large heliocentric distances. 

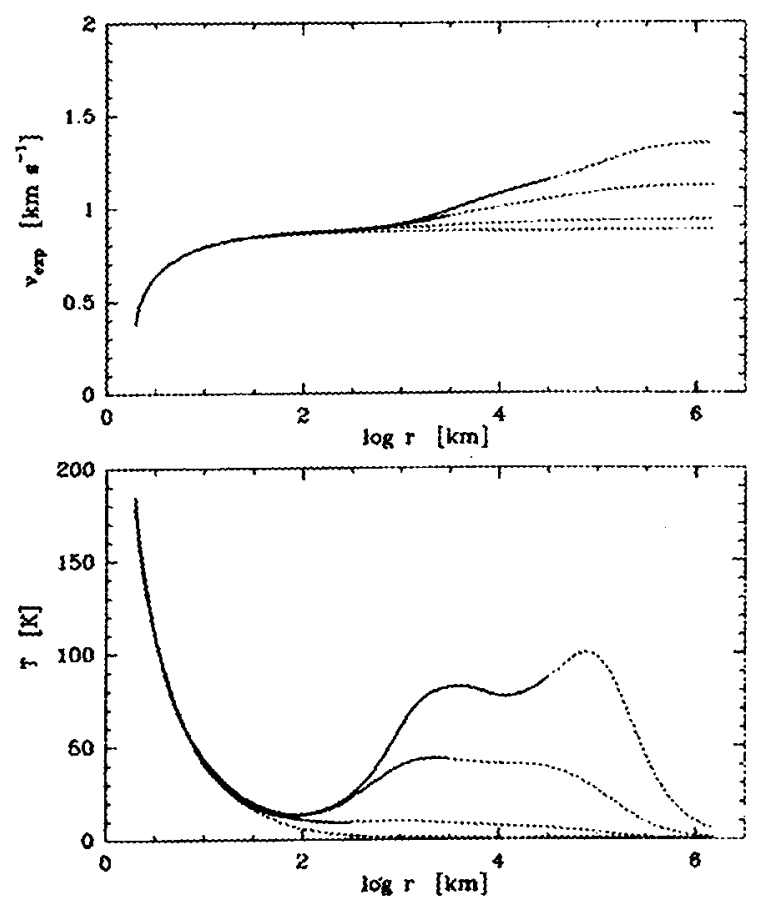

Figure 1. Coma outflow velocities and kinetic temperatures for different gas production rates. Shown are outflow velocities (above) and gas kinetic temperatures (below) for model calculations by Bockelée-Morvan and Crovisier (1987a). In each panel the models from bottom to top correspond to gas production rates of $10^{27}, 10^{28}, 10^{29}$ and $10^{30} \mathrm{~s}^{-1}$. The clear trend for increasing outflow speed and kinetic temperature is apparent. Comet Hale-Bopp at a heliocentric distance of $1 \mathrm{AU}$ had a production rate of $10^{31} \mathrm{~s}^{-1}$.

\section{Models vs. Data for the Coma of Hale-Bopp}

For the First International Conference on Comet Hale-Bopp held in Tenerife in February, 1998, model calculations by Combi et al. (1999b) showed general agreement with the preliminary versions of several sets of observational results available at the time. Figures 6 and 7 show the gas kinetic temperatures and Doppler line widths determined from mm-wavelength radio observations of a number of parent molecule gas species (Biver et al., 2002). Also shown are power-law fits to the heliocentric distance dependencies of each before and after perihelion. These figures contain results which are an update to those presented at the first Tenerife conference (Biver et al., 1999), however the results are essentially similar.

The models of Combi et al. (1999b) shown in Figure 8 were calculated using a relatively simple 1-D spherical steady-state single-fluid hydrodynamic calculation where the heating rate, owing to photochemical heating, is modified iteratively according to the results of a Monte Carlo calculation for photochemical heating via collisions with superthermal H atoms (Combi and Smyth, 1988a; Combi, 

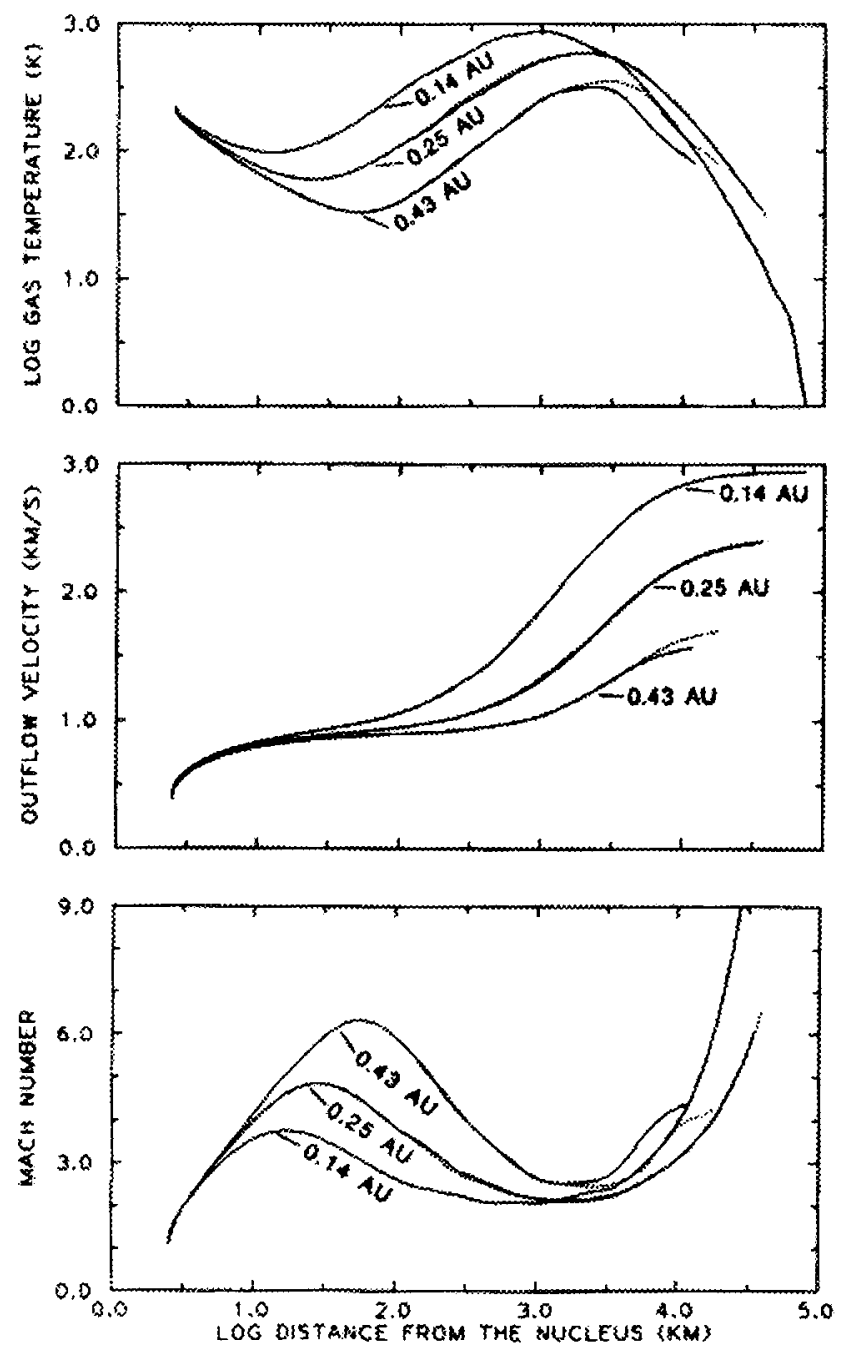

Figure 2. Coupled gas-dynamics Monte Carlo particle trajectory model calculations for comet Kohoutek at different heliocentric distances. Shown from top to bottom are plots of the gas kinetic temperature, outflow velocity and Mach number for a range of heliocentric distances from the paper by Combi and Smyth (1988b). The solid lines are for postperihelion times and the dashed lines are for preperihelion where the gas production rates were $60 \%$ larger at $0.43 \mathrm{AU}$.

1989). Details about the parameters assumed for this hybrid hydrodynamic/Monte Carlo calculation were given by Combi et al. (1999b). As discussed by them, these calculations were integrated over the same observation geometry and density weighting as sampled by the observations of Biver et al. $(1999,2002)$ to produce predicted variations of outflow speed and gas kinetic temperature as a function of heliocentric distance, $r$. The Biver et al. observations give pre- and post-perihelion variations of temperature of $(116 \pm 11) r^{-1.24 \pm 0.09}$ and $(97 \pm 7) r^{1.07 \pm 0.08}$ in Kelvins, 

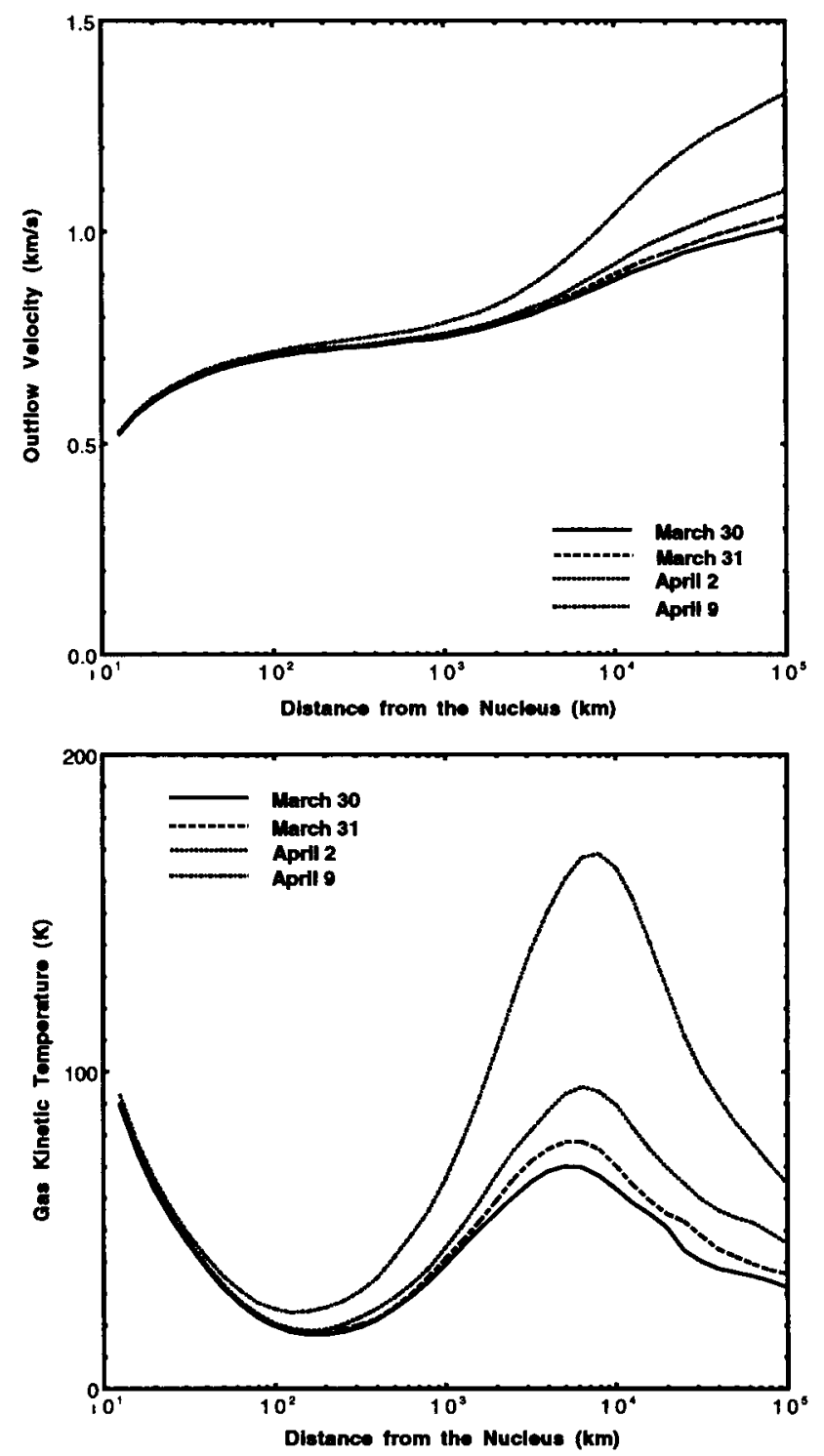

Figure 3. Coupled gas-dynamics Monte Carlo particle trajectory model calculations for comet Hyakutake at different heliocentric distances. Shown are the gas kinetic temperature (above) and outflow velocity (below) for models at a few heliocentric distances for comet Hyakutake by Combi et al. (1999). As in other cases the outflow speeds and temperatures increase with decreasing heliocentric distance and increasing gas production rate. The outflow speed distributions from these models reproduced the observed high spectral resolution line profiles of $\mathrm{C}_{2}, \mathrm{CN}, \mathrm{NH}_{2}, \mathrm{O}\left({ }^{1} \mathrm{D}\right)$ and $\mathrm{H}$ Balmer- $\alpha$. 


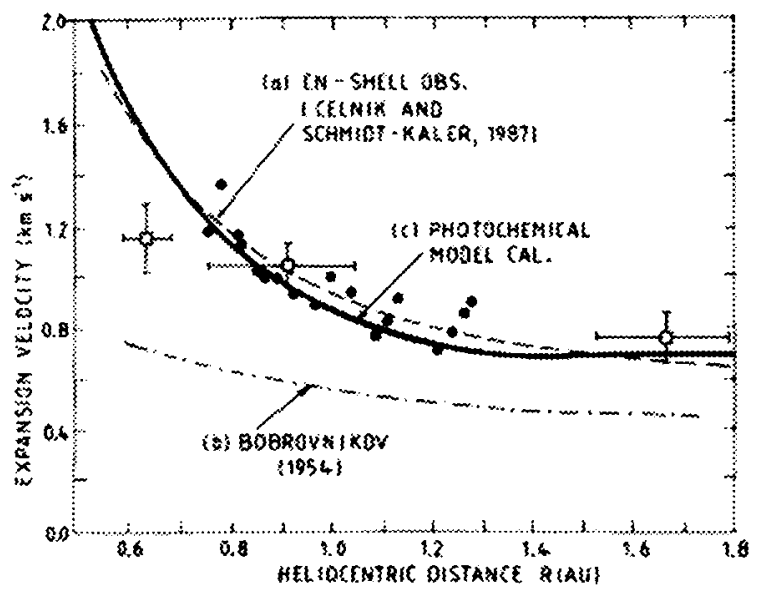

Figure 4. Model-observation comparison for outflow speed in comet 1P/Halley. Shown are models by Ip (1989) for the outflow speed at different heliocentric distances compared with the expansion velocities of $C N$ shells.

respectively, whereas the models for the given parameters yielded $(114) r^{-1.6}$ and $(102) r^{-1.6}$. The Biver et al. observations give pre- and post-perihelion variations of line width of $(1.18 \pm 0.18) r^{-0.44 \pm 0.02}$ and $(1.086 \pm 0.22) r^{-0.04 \pm 0.02}$ in $\mathrm{km} \mathrm{s}^{-1}$, respectively, whereas the models for the given parameter yielded $(1.01) r^{-} 0.32$ and (1.02) $r^{-0.34}$. Considering the fact that laws for the observations were derived with a single temperature and single velocity coma model and that line width is only an approximation to the average outflow speed anyway, the agreement between the observations is quite remarkable.

Colom et al. (1999) presented the results of high velocity resolution 18-mm observations of $\mathrm{OH}$ in comet Hale-Bopp, necessarily using a much larger aperture (half-power beam with of 2.5 by 19 arc minutes) than the observations of Biver et al. (1999, 2002). In addition to extracting $\mathrm{OH}$ (and by implication $\mathrm{H}_{2} \mathrm{O}$ ) production rates, they provided information about the expansion velocity of the coma. Their calculation assumes that $\mathrm{OH}$ radicals are produced upon photodissociation of parent water with a velocity of $1.05 \mathrm{~km} \mathrm{~s}^{-1}$ from the moving center of mass of the water molecules resulting from the excess energy between the dissociating photon and the binding energy holding the $\mathrm{OH}$ and the $\mathrm{H}$ together. The Doppler broadened line of $\mathrm{OH}$ is the result of the convolution of parent water outflow in the coma and this excess velocity. They found that they could understand the line width for HaleBopp near perihelion for an expansion of the water coma of $2.2 \mathrm{~km} \mathrm{~s}^{-1}$. When the comet was at a heliocentric distance of $3 \mathrm{AU}$ before perihelion, the water outflow speed was only $0.7 \mathrm{~km} \mathrm{~s}^{-1}$. The observation beam covers projected distances from the nucleus of several times $10^{5} \mathrm{~km}$. The model calculations in Figure 8 in fact indicate outflow speeds in excess of $2 \mathrm{~km} \mathrm{~s}^{-1}$ for distances larger than $10^{5} \mathrm{~km}$ and heliocentric distance of $1 \mathrm{AU}$ (i.e., near perihelion) and outflow speeds of about 0.7 $\mathrm{km} \mathrm{s}^{-1}$ for heliocentric distances $3 \mathrm{AU}$. 

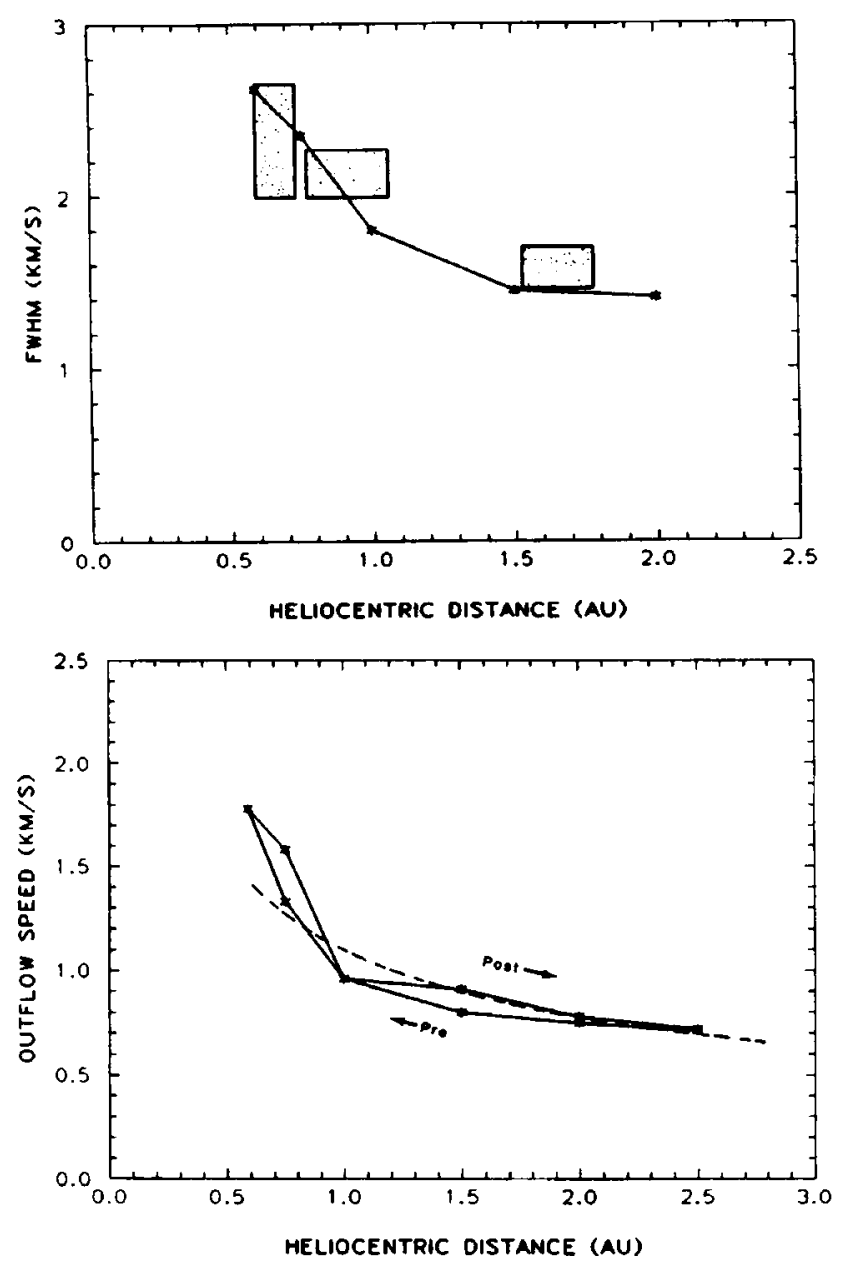

Figure 5. Model-observation comparison for outflow speed in comet 1P/Halley. Shown above is a comparison of the Doppler line widths deduced from the model compared with measurements of line widths of HCN by Schloerb et al. (1987). Shown below are models by Combi (1989) for the outflow speed at different heliocentric distances. Also shown below is an approximation to the variation given by the power law $1.1 r^{-1 / 2} \mathrm{~km} \mathrm{~s}^{-1}$, where $r$ is the heliocentric distance.

An important aspect of these observations, as compared with the smaller aperture observations of Biver et al. is that they demonstrate the large increase in outflow speed, both with decreasing heliocentric distance and with increasing distance within the coma. Both of these properties are consistent with model calculations. From the standpoint of interpreting observations using common simple models (e.g., single-velocity Haser or vectorial models), it means that the outflow speed to use is the average one relevant for the spatial scale of the observations in question. 


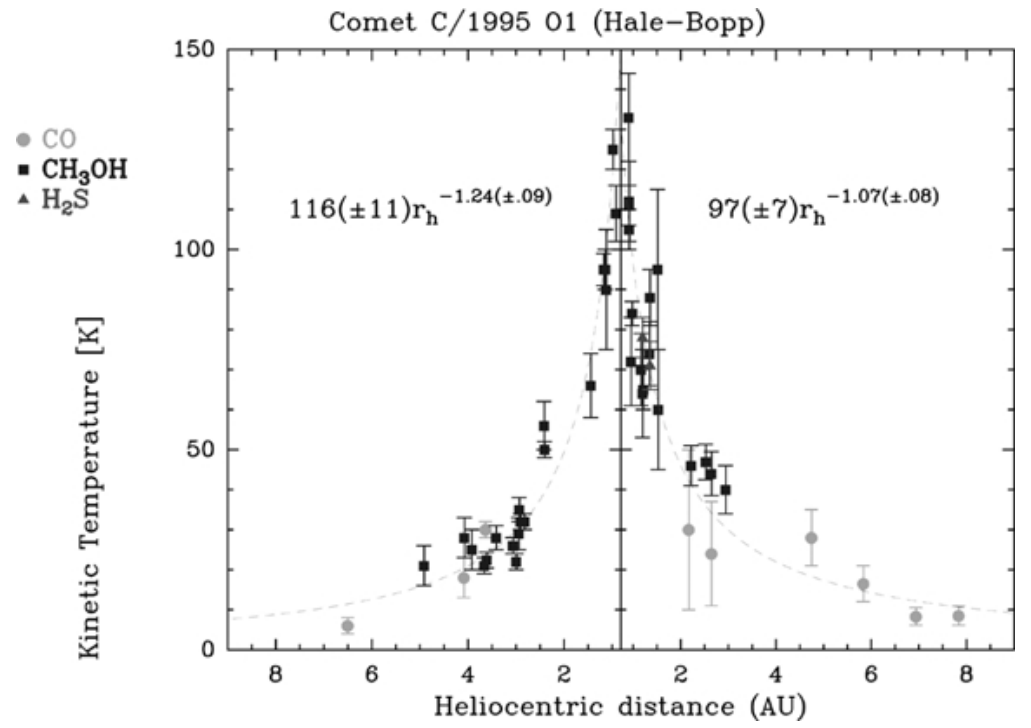

Figure 6. Gas kinetic temperatures from radio observations of comet Hale-Bopp (1995 O1). Shown are gas kinetic temperatures derived from the radio observations of Biver et al. (2002). The effective aperture size covers distances from the nucleus of $1-3 \times 10^{4} \mathrm{~km}$.

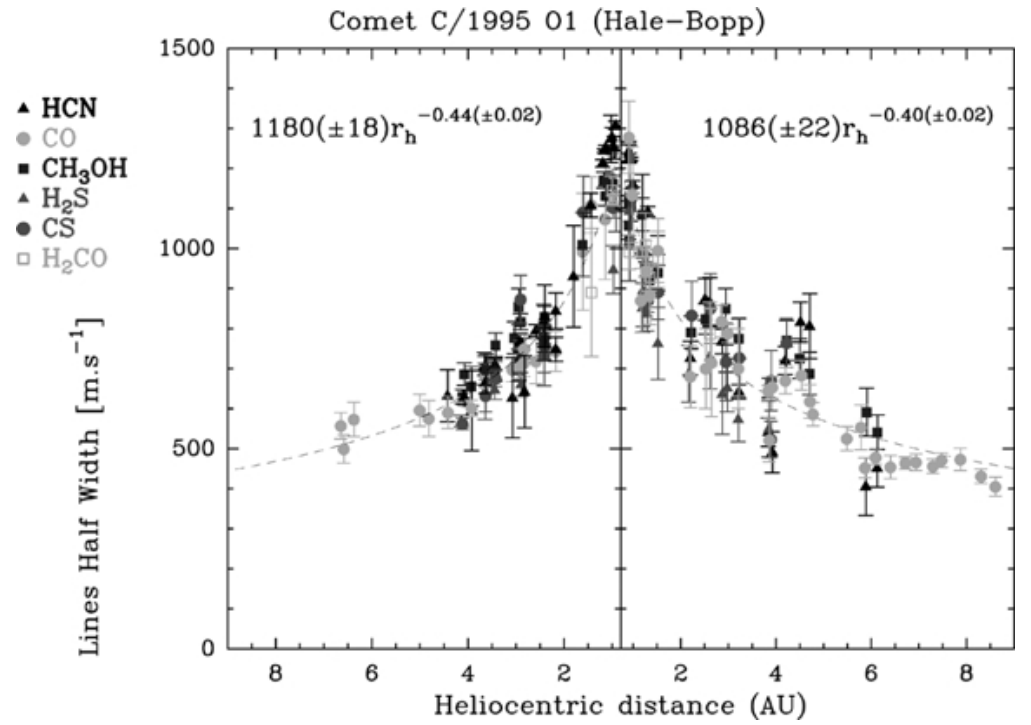

Figure 7. Doppler line widths from radio observations of comet Hale-Bopp (1995 O1). Shown are the Doppler line widths derived from the radio observations of Biver et al. (2002). The line-widths and their variation provide some approximation to the outflow speed within the region covered by the aperture $\left(1-3 \times 10^{4} \mathrm{~km}\right)$ but are not exactly the same. See Figures $5 \mathrm{a}$ and $\mathrm{b}$ and the discussion by Bockelée-Morvan et al. (1990) regarding line shapes and outflow speeds in models. 


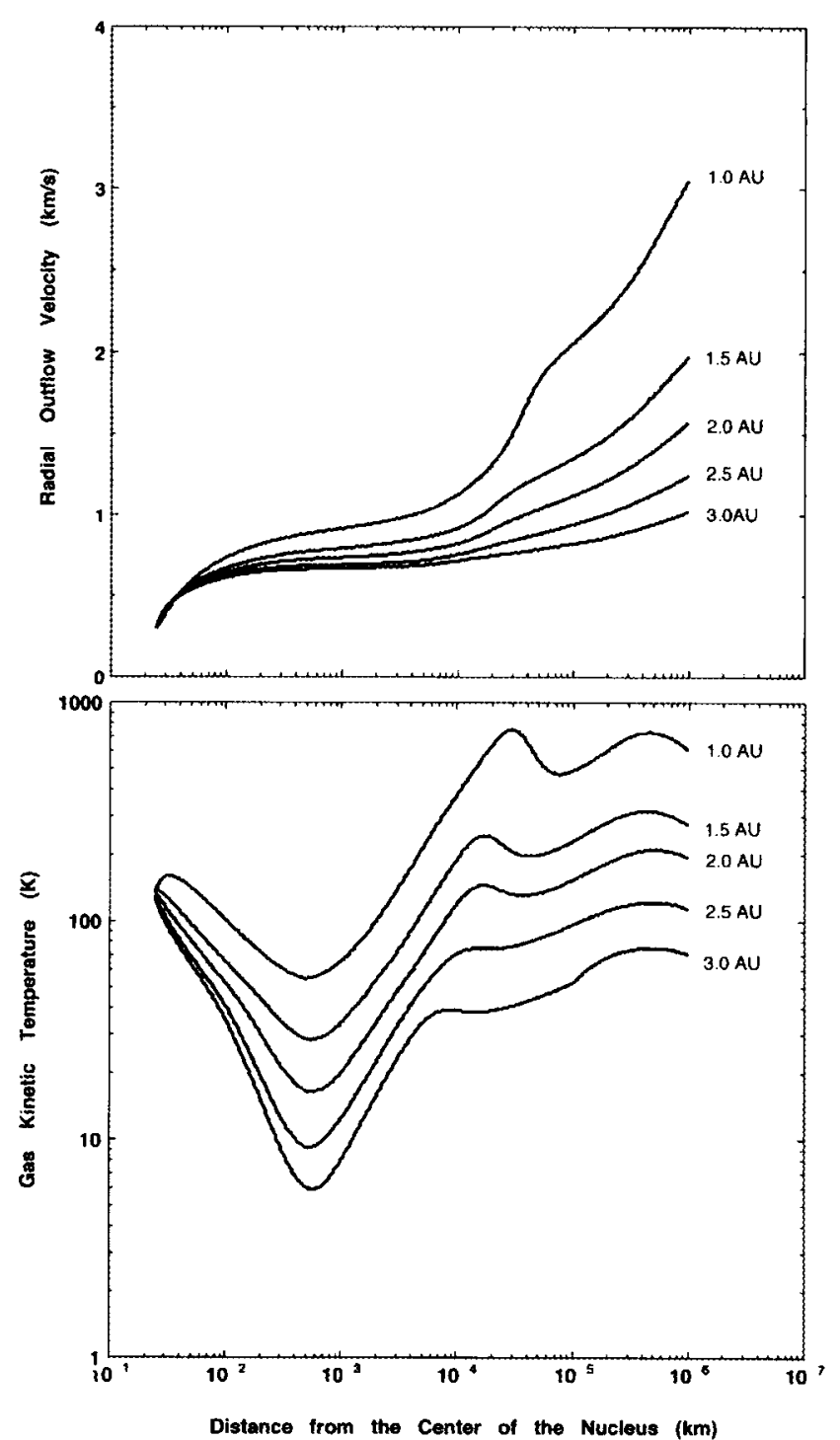

Figure 8. Models for the gas outflow velocity and gas kinetic temperatures in comet Hale-Bopp (1995 O1). Shown are the model calculations by Combi et al. (1999b) from a 1-D spherical hybrid hydrodynamic/Monte Carlo calculation for the coma of comet Hale-Bopp at a range of heliocentric distances.

More recent ground-based observations of propagating $\mathrm{CN}$ shells in comets Hale-Bopp (D. G. Schleicher, private communication) and Hyakutake (Woodney and Schleicher, presentation at the IAU Colloquium 186, Tenerife, Jan. 2002) paint a similar picture. For observations taken near perihelion $\mathrm{CN}$ shells at de-projected distances from the nucleus of 1 to $6 \times 10^{4} \mathrm{~km}$ from the nucleus, the gas velocities were increasing and had a mean value of $1.3 \mathrm{~km} \mathrm{~s}^{-1}$. Again this is in reasonable 
agreement with the model calculations in Figure 8. This is in contrast to observations of comet Hyakutake, which is a Halley-class comet, and where velocities were about $0.8 \mathrm{~km} \mathrm{~s}^{-1}$, similar to comparable model calculations for that comet. Again see Figure 3.

Harris et al. (2002) have presented very wide-field Schmidt-telescope images of the $\mathrm{OH}$ coma in comet Hale-Bopp, extending to distances of $\sim 10^{6} \mathrm{~km}$. They find that they can generally explain the spatial distribution of $\mathrm{OH}$ with a Haserlike model where they increase the outflow speed from less than $1 \mathrm{~km} \mathrm{~s}^{-1}$ in the innermost coma to as large as $3 \mathrm{~km} \mathrm{~s}^{-1}$ at nearly $10^{6} \mathrm{~km}$. Such a picture is again consistent with the other observations and with the model calculations.

Hydrogen atoms, most of which are produced in the photodissociation chain of water and $\mathrm{OH}$, present the inverse picture for velocity as seen in the heavy species in a big comet like Hale-Bopp. The increased speeds seen in the heavy species result from photochemical heating which comes mostly (but not exclusively) at the expense of the superthermal $\mathrm{H}$ atoms produced by the photodissociation of water.

The basic picture of photodissociation production of $\mathrm{H}$ atoms by $\mathrm{H}_{2} \mathrm{O}$ and $\mathrm{OH}$ was first understood quantitatively with the early space ultraviolet measurements of comets in the 1970s (Bertaux and Blamont, 1970; Bertaux et al., 1973; Keller and Meier, 1976). The speed distribution of $\mathrm{H}$ atoms in typical comets (i.e., much less productive than Hale-Bopp) is dominated by an $18-20 \mathrm{~km} \mathrm{~s}^{-1}$ component from the main branch water dissociation $(\mathrm{OH}+\mathrm{H})$ and an $8 \mathrm{~km} \mathrm{~s}^{-1}$ component from the main predissociation branch of $\mathrm{OH}$. Modelling the shape of the coma of comet Kohoutek lead Keller and Meier (1976) to find that in addition to the 8 and $20 \mathrm{~km}$ $\mathrm{s}^{-1}$ components a small low-speed component $\left(4 \mathrm{~km} \mathrm{~s}^{-1}\right)$ was also required, and that more of it was required when the comet was at a smaller heliocentric distance. They concluded that this low speed component was due to thermalized $\mathrm{H}$ atoms. Subsequent detailed physical modelling (Combi and Smyth, 1988b) applied to the same data showed that they were correct.

For comet Hale-Bopp collisional thermalization of $\mathrm{H}$ atoms is quite dominant. The SWAN (Solar Wind Anisotropies) instrument on the Solar and Heliospheric Observatory (SOHO) satellite is designed to make all-sky images of the Lymanalpha emission from interstellar $\mathrm{H}$ atoms as they travel through the solar system and interact with the solar wind. Because the brightness of the typical interplanetary Lyman-alpha emission is of the order of 500 to 1000 Rayleighs, such a camera can easily record the Lyman-alpha coma of even reasonably faint comets (Bertaux et al., 1999; Mäkinen et al., 1999, 2001, 2002). During the first half of 1997, comet Hale-Bopp was by far the dominant object in the Lyman-alpha sky.

Combi et al. (2000) presented 53-days worth of SWAN observations of comet Hale-Bopp during the first half of 1997. They analyzed these data using a 3-D time-dependent Monte Carlo model for the $\mathrm{H}$ atoms which accounts explicitly for production from the outflowing water coma having been heated photochemically (as in Figure 8) and for the multiple collisional thermalization of those atoms. The extent to which the original speed distribution of $\mathrm{H}$ atoms has been slowed 

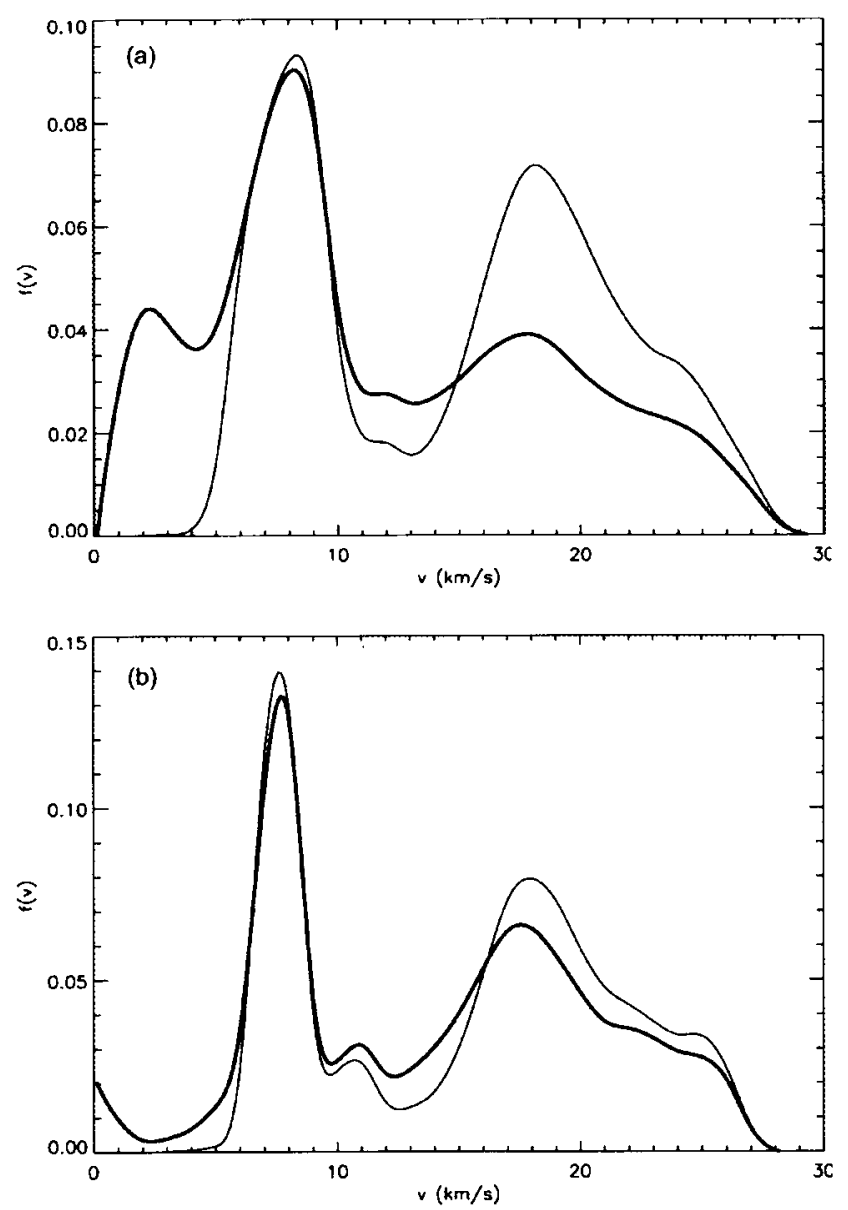

Figure 9. Hydrogen atom speed distribution function. In (a) is a comparison of the effective speed distribution of $\mathrm{H}$ atoms exiting the inner coma with (thick line) and without (thin line) the full physics of partial collisional thermalization from the model for comet Hale-Bopp at perihelion on April 1, 1997. In (b) is a similar comparison except from the model for January 1, 1997 when the comet was $1.75 \mathrm{AU}$ from the sun. Note that for January 1 only a minor modification of the speed distribution results from collisions, whereas the effect is quite important near perihelion.

by collisions is shown in Figure 9. The distribution of $\mathrm{H}$ atom velocities both upon production (i.e., highly superthermal $\mathrm{H}$ atoms before collisions) and after travelling in the coma and colliding with the heavy neutral coma is given for two cases. The first is for SWAN observation on the day of perihelion (April 1, 1997) when the water production rate was $1.02 \times 10^{31} \mathrm{~s}^{-1}$ and the second is for the observation on January 1,1997 , when the water production rate was $2.2 \times 10^{30} \mathrm{~s}^{-1}$.

Also important, though, is the fact that the heliocentric distance at perihelion was $0.91 \mathrm{AU}$ and on January 1 was $1.75 \mathrm{AU}$. This means that all photodissociation and photoionization lifetimes were 3.7 times longer on January 1 than on April 1 . 
The combination of decreased gas density from the factor of 5 decrease in production rate and the increased photochemical lifetime, which produces daughter species at even larger distances from the nucleus dramatically decreases the collisional thermalization of the superthermal $\mathrm{H}$ atoms. As seen in Figure 9, the pre- and post-collision distribution functions were only modestly different on January 1 but much of the highest speed components are removed from the original distribution on April 1. This causes a dramatic decrease in the average speed of $\mathrm{H}$ atoms in the outer coma which contribute to the SWAN images. Using the photochemical distribution for $\mathrm{H}$ atoms without accounting for collisions in a model to extract the water production rate from the SWAN observations would lead to a large overestimate of the production rate by more than $50 \%$. Figure 10 shows a model-data comparison of the full image for the April 1 observation which represents the most severe test of the accuracy of the modelled $\mathrm{H}$ speed distribution. The distortion from rough circularity and the sunward extend of the $\mathrm{H}$ coma is limited by solar radiation pressure acceleration via resonance scattering of the $\mathrm{H}$ atoms by the same solar UV Lyman-alpha photons which enable us to see the coma itself. A model which does not slow the $\mathrm{H}$ atoms significantly produces a much more circular $\mathrm{H}$ coma than observed or than modelled with the correct velocity distribution. Finally, Figure 11 shows an updated comparison of water production rates from the SWAN observations, beyond that in Combi et al. (2000), to include some more recently published observations.

More recently, Morgenthaler et al. (2002) have presented very high spectral resolution line profiles of the hydrogen Balmer-alpha line profile of comet HaleBopp obtained with a scanning Fabry-Perot etalon. In a preliminary analysis of their data they find a line profile that is narrower than comparable observations made of comet Halley (Smyth et al., 1994). They conclude that the narrow line profiles are evidence for more slowly outflowing $\mathrm{H}$ atoms produced by more collisions with the outflowing heavy-molecule gas coma in Hale-Bopp as described above.

\section{Summary}

The large gas production rate of comet Hale-Bopp had important consequences on the dynamical state of the coma. This was especially true for two to three months around perihelion, where the combination of large gas densities and short dissociation lifetimes produced a much larger than normal amount of superthermal atoms and radicals within the collision-dominated coma. This first heats the coma resulting in increased gas kinetic temperature and then in larger gas outflow speeds than those found in typical so-called bright comets at heliocentric distances near 1 AU, e.g., 1P/Halley and Hyakutake (1996 B2). Observations of faster outflow speeds for heavy molecules (e.g., $\mathrm{OH}$ and $\mathrm{CN}$ ), as well as slower outflow speeds for atomic $\mathrm{H}$ are consistent with this picture. 


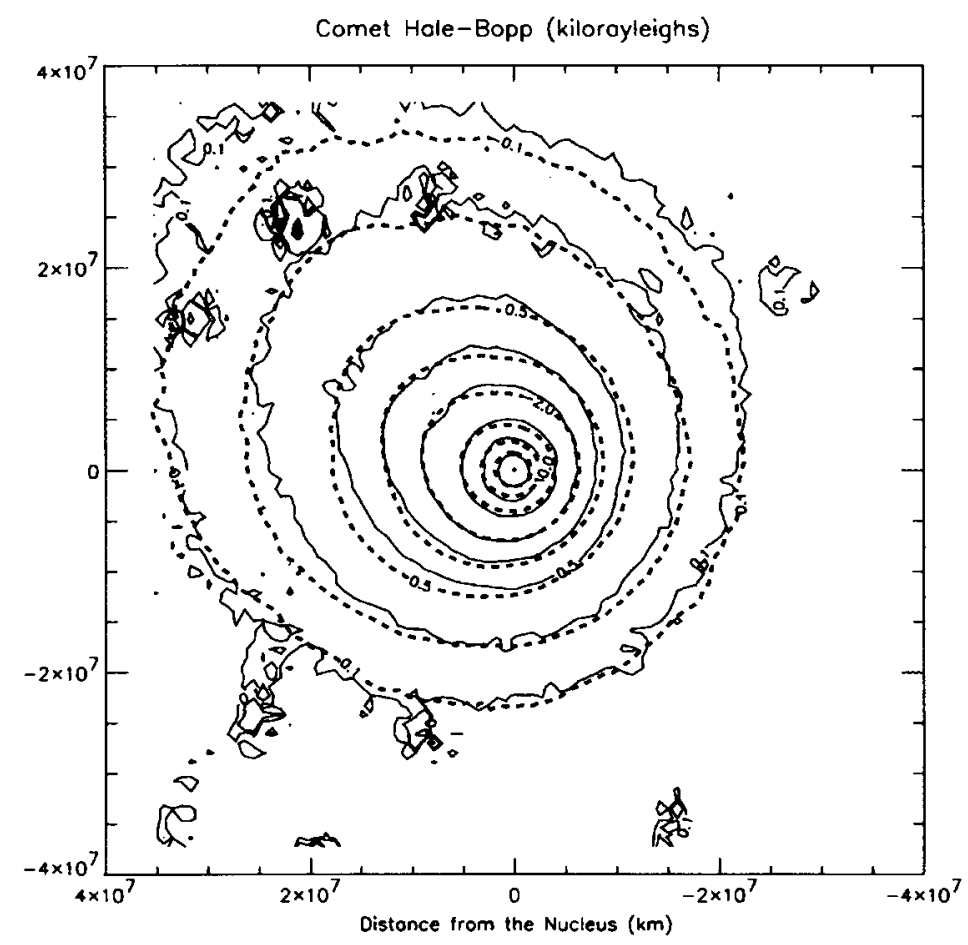

Figure 10. Model-data comparison of $\mathrm{H} \mathrm{Ly}-\alpha$ isophote contours on 1 April 1997. Contours of brightness in kilorayleighs for the SWAN data (solid lines) are compared with those from a model (dashed lines). The model calculation (see Combi and Smyth, 1988b; Combi et al., 2000) is able to reproduce both the shape and overall radial gradient of the coma. The observed shape is produced by a combination of $\mathrm{H}$ atom velocity distribution from photodissociation and partial thermalization, radiation pressure acceleration from solar fluorescent scattering, and the relative orbital motions of the nucleus and $\mathrm{H}$ atoms.

The most important implication of this is that when extracting gas production rates from limited-aperture observations of comets it is necessary to account for the appropriate outflow velocity for that species in the given aperture. The canonical 1 $\mathrm{km} \mathrm{s}^{-1}$ could lead to errors in the gas production rates by factors of 2 to 3 . More seriously, these errors would lead to incorrect scale lengths and to false heliocentric distance dependencies because only the outflow speeds near perihelion are seriously affected, while those at larger distances of $1.7 \mathrm{AU}$ and larger are nominal. In general the outflow velocity can vary from species to species, with the heliocentric distance of the comet, and with the distance from the nucleus which contributes to the observation. One example of this is the set of small aperture mm radio observations of Biver et al. (2002), which found, and used, an appropriate velocity law near $1 \mathrm{~km} \mathrm{~s}^{-1}$ at $1 \mathrm{AU}$ and near perihelion $1.2 \mathrm{~km} \mathrm{~s}^{-1}$. Contemporaneous observations of the 18-mm emission of $\mathrm{OH}$ by Colom et al. (1999) used a much larger aperture and had to account appropriately for much larger velocities of $\sim 2.2 \mathrm{~km} \mathrm{~s}^{-1}$. This was also true for wide-field ground-based imaging of $\mathrm{OH}$ by Harris et al. (2002). 

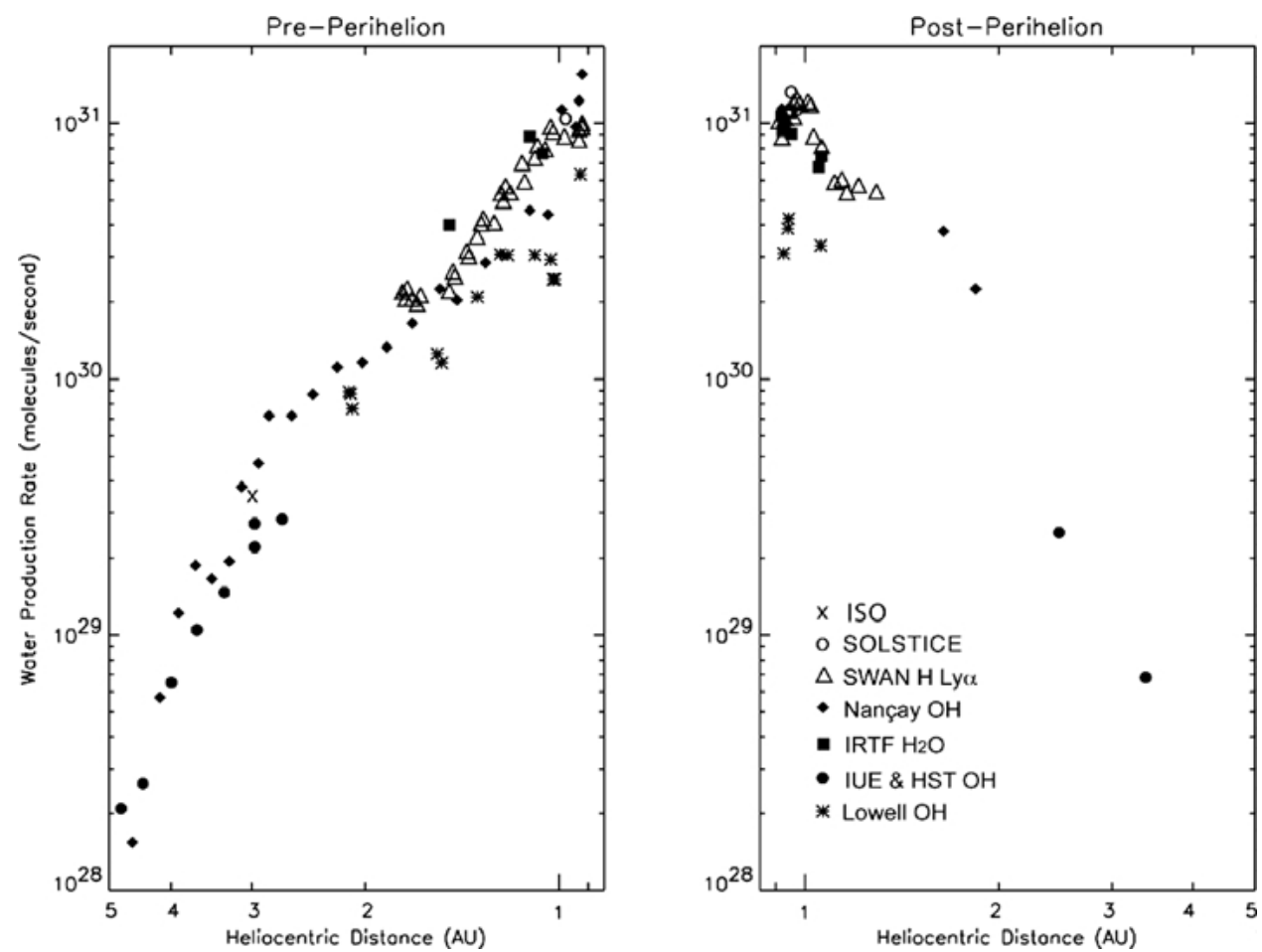

Figure 11. Comparison of water production rates in comet Hale-Bopp. The water production rates of Hale-Bopp determined from the SWAN observations of H Ly- $\alpha$ are compared with those determined from ISO observations by Crovisier et al. (1997), SOLSTICE observations by Woods et al. (2000), IRTF observations of $\mathrm{H}_{2} \mathrm{O}$ by Dello Russo et al. (1999) and various observations of $\mathrm{OH}$ by Colom et al. (1999) from Nançay, Weaver et al. (1999) with HST and IUE, and Schleicher et al. (1997, 1999) from ground-based photometry at Lowell Observatory.

More generally, coma outflow velocities also affect molecular scale lengths which are a combination of velocity and lifetime. Incorrect use of these can lead to even larger systematic errors in extraction of production rates from observations

\section{Acknowledgements}

Support for this work from grant NAG5-8942 from the NASA Planetary Atmospheres program is gratefully acknowledged. The author also thanks N. Biver, D. Schleicher, T. Farnham, L. Woodney, W. Harris, and J. Morgenthaler for sharing their observational results before publication. 


\section{References}

Bertaux, J.-L. and Blamont., J.: 1970, Comput. Rend. Acad. Sci. 270, 1578-1584.

Bertaux, J.-L., Blamont, J., and Festou, M. C.: 1973, Astron. Astrophys. 25, 415-430.

Bertaux, J.-L., Costa, J., Mäkinen, T., Quémerais, E., Lallement, R., Kyrölä, E., and Schmidt, W.: 1999, Planet. Space Sci. 47, 725-733.

Biver, N. and 22 colleagues: 1997/1999, Earth Moon Planets 78, 5-11.

Biver, N. and 23 colleagues: 2002, these proceedings.

Bockelée-Morvan, D. and Crovisier, J.: 1987a, in Diversity and Similarity of Comets ESA SP-278, pp. 235-240.

Bockelée-Morvan, D. and Crovisier, J.: 1987b, Astron. Astrophys. 187, 425-430.

Bockelée-Morvan, D. and Crovisier, J.: 2002, these proceedings.

Bockelée-Morvan, D., Crovisier, J., and Gérard, E.: 1990, Astron. Astrophys. 238, 382-400.

Chamberlain, J.: 1962, Planetary Coronae and Atmospheric Evaporation, Planet. Space Sci. 11, 901960.

Colom, P., Gérard, E. Crovisier, J., Bockelée-Morvan, D., Biver, N., and Rauer, H.: 1997/1999, Earth Moon Planets 78, 37-43.

Combi, M. R.: 1989, Icarus 81, 41-50.

Combi, M. R.: 1996, Icarus 123, 207-226.

Combi, M. R. and Smyth, W. H.: 1988a, Astrophys. J. 327, 1026-1043.

Combi, M. R. and Smyth, W. H.: 1988b, Astrophys. J. 327, 1044-1059.

Combi, M. R., Cochran, A. L., Cochran, W. D., Lambert, D. L., and Johns-Krull, C. M.: 1999a, Astrophys. J. 512, 861-968.

Combi, M. R., Kabin, K., DeZeeuw, D. L., Gombosi, T. I., and Powell, K. G.: 1997/1999b, Earth Moon Planets 79, 275-306.

Combi, M. R., Reinard, A., Bertaux, J.-L., Quémerais, E., and Mäkinen, T.: 2000, Icarus 144, 191202.

Crifo, J. F.: 1989, Astron. Astrophys. 223, 365-368.

Crovisier, J.: 1984, Astron. Astrophys. 130, 361-372.

Crovisier, J.: 1989, Astron. Astrophys. 213, 459-464.

Dello Russo, N., Mumma, M. J., DiSanti, M. A., Magee-Sauer, K., Novak, R., and Rettig T. A.: 2000, Icarus 143, 324-337.

Delsemme, A. H. and Miller, D. C.: 1970, Planet. Space Sci. 19, 1229-1257.

Gombosi, T. I., Cravens, T. E., and Nagy, A. F.: 1985, Astrophys. J. 293, 328-341.

Gombosi, T. I., Nagy, A. F., and Cravens, T. E.: 1986, Rev. Geophys. 24, 667-700.

Harris, W. M., Morgenthaler, J. P., Scherb, F., Anderson, C., and Oliversen, R.: 2002, these proceedings.

Huebner, W. F. and Keady, J. J.: 1983, in T. I. Gombosi (ed.), Cometary Exploration, Central Research Institute for Physics, Budapest, Hungary, pp. 165-183.

Huebner, W. F. Keady, J. J., and Lyon, S. P.: 1992, Astrophys. Space Sci. 195, 1-289.

Ip, W.-H.: 1989, Astrophys. J. 346, 475-480.

Keller, H. U. and Meier, R. R.: 1976, Astron. Astrophys. 52, 273-281.

Kitamura, Y.: 1986, Icarus 66, 241-257.

Lovell, A. J.; Schloerb, F. P., Dickens, J. E., De Vries, C. H., Senay, M. C., and Irvine, W. M.: 1998, Astrophys. J. 497, L117-L121.

Mäkinen, T., Bertaux, J.-L., Laakso, H., Pukkinen, T. I., Schmidt, W., Kyrölä, E., Summanen, T., Quémerais, E., and Lallement, R.: 2000, Nature 405, 321-324.

Mäkinen, T., Bertaux, J.-L., Pukkinen, T. I., Schmidt, W., Kyrölä, E., Summanen, T., Quémerais, E., and Lallement, R.: 2001, Astron. Astrophys. 368, 292-297.

Mäkinen, T., Bertaux, J.-L., Combi, M. R., and Quémerais, E.: 2001, Science 292, 1326-1329.

Marconi, M. L. and Mendis, D. A.: 1983, Astrophys. J. 273, 381-396. 
Morgenthaler, J. P., Harris, W. M., Scherb, F., Doane, N. E., and Oliversen, R. J.: 2002, these proceedings.

Schleicher, D. G., Lederer, S. M., Millis, R. L., and Farnham, T. L.: 1997, Science 275, 1913-1915.

Skorov, Y. V. and Rickman, H.: 1998, Planet. Space Sci. 46, 975-996.

Stern, S. A., Slater, D. C., Festou, M. C., Parker, J. Wm., Gladstone, G. R., A'Hearn, M.F., and Wilkinson, E.: 2000, Astrophys. J. 544, L169-L172.

Weaver, H. A., Feldman, P. D., A’Hearn, M. F., Arpigny, C., Brandt, J. C., and Stern, S. A.: 1999, Icarus 141, 1-12.

Whipple, F. L. and Huebner, W. F.: 1976, Annu. Rev. Astron. Astrophys. 14, 143-172.

Woods, T. N., Feldman, P. D., and Rottman, G. J.: 2000, Icarus 144, 182-186.

Wu, C. Y. R. and Chen, F. Z.: 1993, J. Geophys. Res. 98, 7415-7435. 